\title{
可见光诱导重氮化合物产生卡宾及其官能化反应
}

\author{
蔡宝贵宣 俊* \\ (安徽大学化学化工学院 无机-有机杂化功能材料化学安徽省重点实验室 合肥 230601)
}

\begin{abstract}
摘要 卡宾是有机合成中的重要中间体. 在过去的几十年中, 过渡金属催化的卡宾转移反应取得了显著发展. 近年来, 可见光促进的重氮化合物通过游离态卡宾的转化也开始兴起. 该类反应仅需要使用可见光作为唯一能源, 符合绿色 化学理念. 自 2018 年 Davies 课题组和周否课题组分别独立报道了芳基重氮酯在可见光照射下光解生成游离态卡宾物 种以来, 光促重氮化合物的转化反应受到了越来越多科研工作者的关注, 也催生了许多绿色高效的有机合成新反应. 在前人工作基础上, 进一步完善了该领域的最新进展, 综述了近期可见光照射下重氮化合物的新转化反应和国内学 者的贡献，展望了该领域的未来发展方向和所面临的挑战.
\end{abstract}

关键词 可见光; 重氮化合物; 卡宾; 绿色化学

\section{Visible Light-Promoted Transformation of Diazo Compounds via the Formation of Free Carbene as Key Intermediate}

\author{
Cai, Baogui Xuan, Jun* \\ (Anhui Province Key Laboratory of Chemistry for Inorganic/Organic Hybrid Functionalized Materials, \\ College of Chemistry \& Chemical Engineering, Anhui University, Hefei 230601)
}

\begin{abstract}
Carbene is one of the most important synthetic intermediates in organic synthesis. In the past few decades, transition-metal catalyzed carbene transfer reactions have made remarkable development. Recently, visible light-promoted transformation of diazo compounds through the formation of free carbene as key intermediate begun to rise. The reaction only need visible light as the sole energy source which meets the concept of green chemistry. Since the pioneering works developed by the groups of Diaves and Zhou, photo-promoted transformation of diazo compounds has attracted more and more attentions. On the basis of previous work, the latest progress in this field is further improved, which mainly focuses on the recent new transformation reactions of diazo compounds under visible light irradiation and the contributions reported from Chinese research group. The future development direction, as well as challenges in this field is prospected.

Keywords visible light; diazo compound; carbene; green chemistry
\end{abstract}

重氮化合物是有机化学中一类重要合成中间体. 早 在 19 世纪中期, Meerwein 等 ${ }^{[1]}$ 就发现了重氮甲烷可以在 紫外光照射下进行光解, 产生的卡宾中间体具有很高的 反应活性，可与乙醚分子进行 $\mathrm{C}-\mathrm{H}$ 官能化反应. 随后, 光促卡宾转移反应的一些其他合成应用也相继被报 道 ${ }^{[2]}$, 但重氮化合物在紫外光条件下进行光解产生的游 离态卡宾选择性较低, 副反应多, 制约了该类反应在合 成有机化学中的应用. 1961 年, Skell 和 Etter ${ }^{[3]}$ 发现重氮 乙酸乙酯在铜催化的条件下能够与环已烯进行环丙烷 化反应, 其选择性远远高于紫外光诱导的卡宾转移过
程. 在随后的几十年里, 过渡金属催化条件下的卡宾转 移反应取得了跨越式的发展, 通过配体调控, 能够实现 对反应活性以及选择性的精准控制. 金属催化的卡宾转 移反应也是当今被认为合成效率最高、应用范围最广的 有机合成方法之一 ${ }^{[4]}$.

随着可见光催化技术的不断发展 ${ }^{[5]}$, Davies 课题组 和周磊课题组 ${ }^{[6]}$ 在 2018 年分别报道了可见光诱导下芳 基重氮酯通过游离态卡宾中间体的转化. 如 Scheme 1 所示, 对不同类型重氮化合物的光学性质研究发现, Diels-Alder 类型重氮化合物在可见光区域有非常明显

* Corresponding author. E-mail: xuanjun@ahu.edu.cn

Received September 26, 2021; revised October 20, 2021; published online October 28, 2021.

Project supported by the National Natural Science Foundation of China (Nos. 21971001, 21702001).

国家自然科学基金(Nos. 21971001, 21702001)资助项目. 
的吸收. 这类重氮化合物在可见光照射下进行光解产生 游离态卡宾, 这也是目前可见光促进重氮转化反应中应 用最多的底物类型. 近期, Koenigs 课题组 ${ }^{[7]}$ 发现, 修饰 后的二芳基重氮化合物也可在蓝色 LED 照射下产生卡 宾物种，随后发生后续的环丙烯化等反应. 近几年来, 由于可见光诱导重氮化合物的转化反应具有操作简单、 无需使用金属催化剂等优势, 以及符合绿色化学等理 念, 吸引了越来越多科研工作者的关注. 同时该类反应 使用能量低的可见光作为光源, 有效避免了一些不必要 的副反应, 使得可见光诱导的卡宾转移反应通常能够以 较高的收率进行.

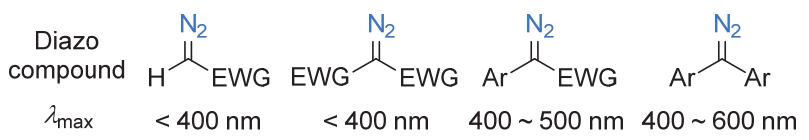

图式 1 不同类型重氮化合物的吸收范围

Scheme 1 Absorption range of diverse diazo compounds

目前, 可见光诱导的重氮化合物通过游离态卡宾中 间体的转化主要被用于环丙烷化反应, $\mathrm{C}-\mathrm{H}$ 键和 $\mathrm{X}-\mathrm{H}$ 键 $(X=N, O, S, S i$ 等)的插入反应以及通过叶立德中间 体进行的转化(例如 Doyle-Kirmse 反应或 SommeletHauser 重排 $)^{[8]}$. 与发展成熟的过渡金属催化相比, 可见 光诱导重氮化合物的转化还处于起步发展阶段. 在该领 域中, Koenigs 课题组和 Gryko 课题组 ${ }^{[9]}$ 相继对相关报道 进行了归纳总结, 但所涉及到的工作大多是 2018 2020 年发表的论文. 另一方面在上述两篇综述中, 中国 学者的贡献并没有得到太多关注. 在此背景下, 本篇综 述将进一步完善该领域的最新进展，以近期可见光照射 下重氮化合物的新转化反应和国内学者的贡献为主, 并 对该领域的未来发展方向和所面临的挑战做出讨论.

\section{1 环丙烷化反应}

环丙烷化是重氮化合物最重要的反应之一. 2018 年, Davies 和 Jurberg 等 ${ }^{[6]}$ 首次报道了可见光诱导下芳基 重氮酯与苯乙烯的环丙烷化反应(Scheme 2). 在蓝色 LED 灯照射下, 芳基重氮酯 $\mathbf{1}$ 光解产生游离态卡宾中间 体 2 , 随后与苯乙烯 $\mathbf{3}$ 发生 $[2+1]$ 环加成反应, 得到环丙 烷化产物 4. 该方法不需要严格的除水操作, 在室温条 件下就能够以高收率和高非对映选择性获得目标产物 4. 此外, 四川大学吴勇团队 ${ }^{[10]}$ 使用腙类化合物作为原 料, 在碱的协助下原位产生重氮化合物, 随后经过光解 产生游离态卡宾, 同样也实现了苯乙烯的环丙烷化反 应.

随后, Koenigs 课题组 ${ }^{[11]}$ 发现, 苯、甲苯、二甲苯等 芳环化合物同样可以捕获可见光促进下芳基重氮酯产 生的游离态卡宾, 成功地实现了芳环的环丙烷化. 受此

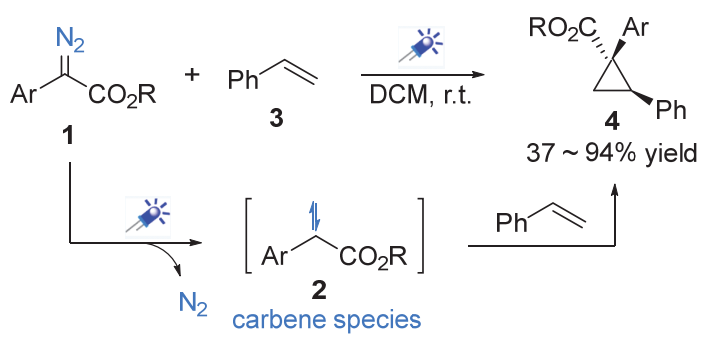

图式 2 可见光诱导芳基重氮酯与苯乙烯的环丙烷化反应 Scheme 2 Visible light induced cyclopropanation of aryldiazoacetates with styrene

工作启发, 常州大学陈新课题组 ${ }^{[12]}$ 利用吲哚酮类重氮 化合物 5 作为卡宾前体，成功实现了卡宾对芳环 6 的环 丙烷化反应(Scheme 3), 以较高收率得到了一系列具有 重要合成价值的螺环氧化吲哚产物 7.
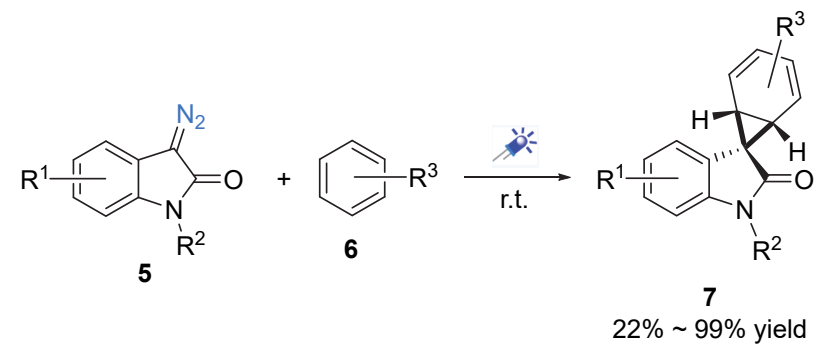

图式 3 可见光诱导吲哚酮类重氮与芳环的环加成反应 Scheme 3 Visible light induced cyclopropanation of diazooxindoles with arenes

高度官能化的杂环环丙烷化合物已被证明是构建 活性药物分子的有效前体 ${ }^{[13]}$. 在 Koenigs 课题组 ${ }^{[14]}$ 前期 的工作基础上, Barham 和 Reiser 等 ${ }^{[15]}$ 通过流动光化学技 术实现了呋喃、吡咯等杂环化合物的环丙烷化反应 (Scheme 4). 值得注意的是, 该反应在低毒性的碳酸二 甲酯中即能进行, 并且与传统的光化学反应相比, 该反 应能够获得更高的反应收率，并且大大缩短了反应时 间. 该策略同时也为日后利用重氮化合物进行工业化生 产奠定了一定的基础.

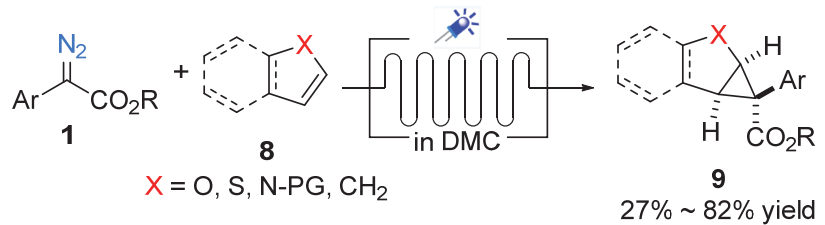

图式 4 连续流动光化学条件下的环丙烷化反应

Scheme 4 Photochemical cyclopropanation under continuous flow conditions

最近, Sen 课题组 ${ }^{[16]}$ 发现芳基重氮酯 $\mathbf{1}$ 与吲哚衍生 物 10 在可见光照射下, 通过药品添加顺序的不同可以 选择性地合成 $\gamma$-咔啉衍生物 11 和螺环吲哚衍生物 12 (Scheme 5). 值得一提的是, $\gamma$-咔啉衍生物 11 在空气中就 
能被氧化得到芳构化产物 13. 机理研究表明, 可见光促 进芳基重氮酯 $\mathbf{1}$ 与吲哚衍生物 $\mathbf{1 0}$ 的环丙烷化是反应的 核心步骤, 随后通过三氟乙酸(TFA)与水合肼添加顺序 的不同可以选择性地以中等至良好的收率合成 11 和 12. 值得注意的是, 如果最后一步使用少量的三氟乙酸, 就 会获得 11 和 12 的等量的混合物.

\section{$2 \mathrm{X}$ - $\mathrm{H}$ 插入反应}

$\mathrm{X}-\mathrm{H}$ 插入是卡宾化学的另一重要分支, 该策略是 构建 $\mathrm{C}-\mathrm{C}$ 键以及 $\mathrm{C}-\mathrm{X}$ 键最有效的方法之一 ${ }^{[17]}$. 在 Davies 和 Jurberg 报道了可见光促进的芳基重氮酯对 $\mathrm{X}-\mathrm{H}$ 的插入反应以前, 这一领域几乎完全由过渡金属 催化的反应主导(Scheme 6). Davies 和 Jurberg ${ }^{[6]}$ 分别对 羧酸 17、胺 19、烷烃 21 、芳环 23 进行了 $X-H$ 插入的 研究, 均能够以中等至良好的收率获得目标产物. 反应 不需要任何催化剂以及添加剂, 并且反应条件温和, 符 合绿色化学的理念. 受此工作启发, 许多其他类型的光 促卡宾中间体对 $\mathrm{X}-\mathrm{H}$ 键的插入反应相继被发展报
道 ${ }^{[18]}$. 值得注意的是, 过渡金属催化和可见光促进的重 氮化合物转化在某些情况下得到了相互补充.

肜醚是一类重要的有机结构单元, 广泛存在于许多 天然产物和活性药物分子中 ${ }^{[19]}$. 然而传统构建肟醚的 方法一般需要额外的添加剂或催化剂, 且原子利用率也 相对较低. 基于此, 我们课题组 ${ }^{[20]}$ 最近报道了可见光促 进下，芳基重氮酯 $\mathbf{1}$ 产生的卡宾对肪 $\mathbf{2 5}$ 的 $\mathrm{O}-\mathrm{H}$ 插入反 应(Scheme 7)，以中等至良好的收率构建相应的肜醚 26.

多组分反应由于具有操作简单，选择性好等特点， 备受有机化学家们的青睐 ${ }^{[21]}$. 通过合理的反应设计, 实 现可见光促进的芳基重氮酯参与的多组分反应无疑具 有很高的研究价值. 由于游离态的卡宾反应活性较高, 其多组分反应的有序进行具有一定挑战性. 基于课题组 前期在光促亚硝基芳烃转化方面的研究基础 ${ }^{[22]}$, 我们 近期实现了芳基重氮酯、亚硝基芳烃与 $\beta$-酩酸酯在可见 光照射下的三组分反应，高产率得到了一系列三取代羟 胺衍生物 29 (Scheme 8) ${ }^{[23]}$. 研究表明, $\beta$-酮酯 27 与 2-亚 硝基吡啶 28 在较短的时间内即可完成亲核加成反应,

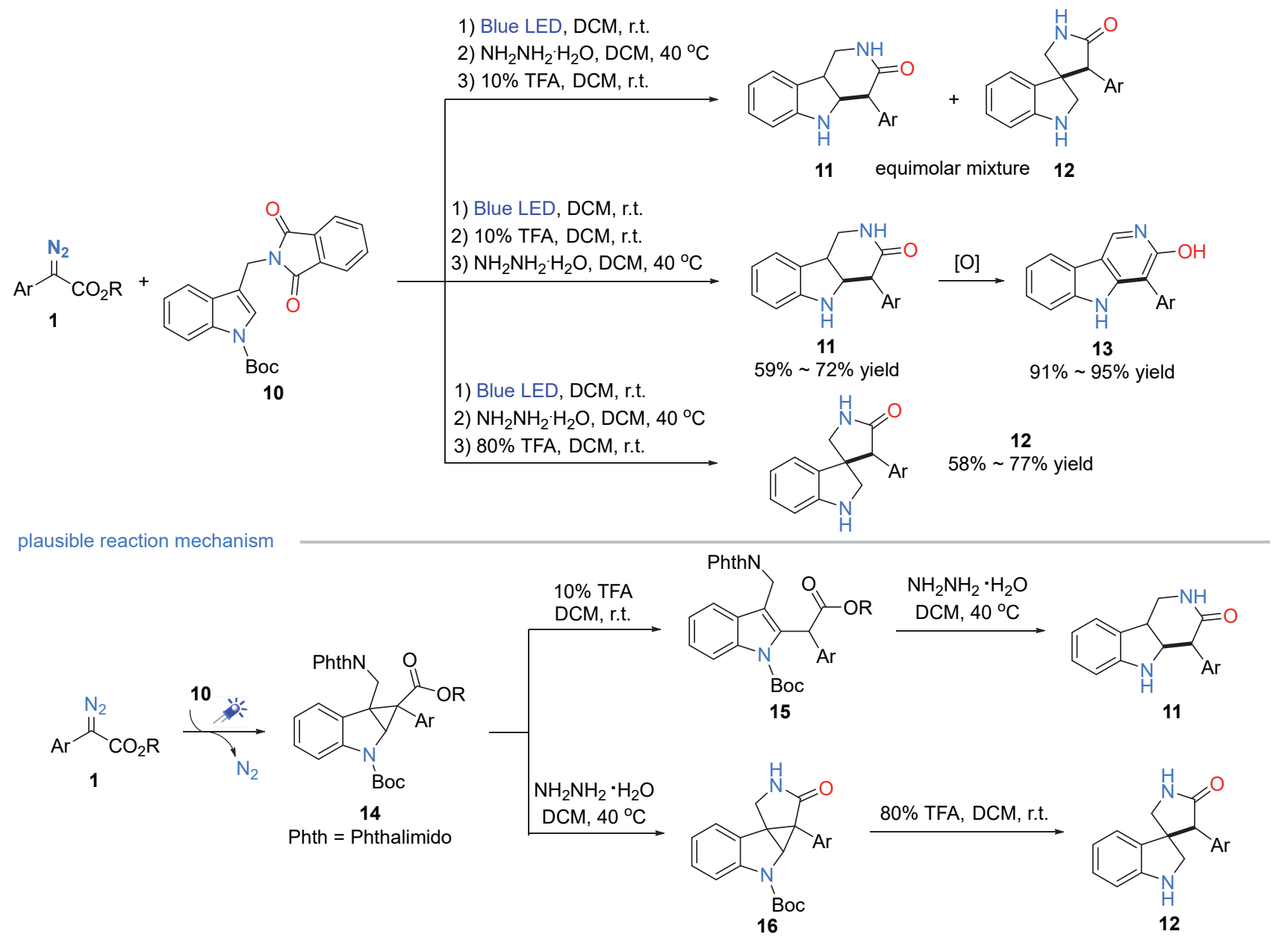

图式 5 可见光促进芳基重氮酯与吲哚衍生物的反应

Scheme 5 Visible light promoted reaction of aryldiazoacetates with indole derivatives 


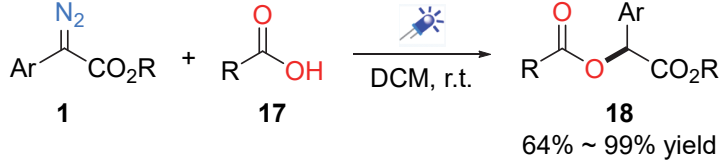<smiles>[R]N[14N]N</smiles><smiles>[R]OC(=O)C([Al])N([R])[R]</smiles>

20 $58 \% \sim 90 \%$ yield<smiles>N#CC(=O)OC1CCCC1</smiles><smiles>[R]OC(=O)C(Br)C1CCNC1</smiles>

22 $47 \% \sim 91 \%$ yield<smiles>[R]OC(=O)C([Al])c1ccccc1</smiles>

图式 6 可见光促进卡宾对 $\mathrm{X}-\mathrm{H}$ 键的插入反应

Scheme 6 Visible light promoted insertion of carbene into $\mathrm{X}$ $\mathrm{H}$ bond

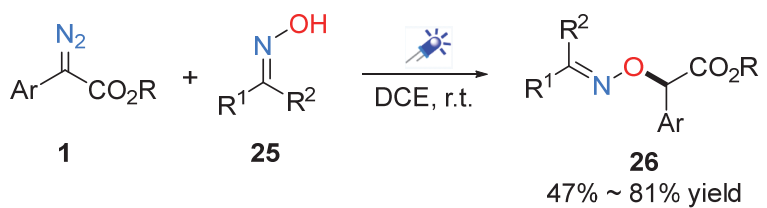

图式 7 可见光促进卡宾对肜的插入反应

Scheme 7 Visible light promoted insertion of carbene into oximes

得到相应的亚硝基-aldol 产物 $\mathbf{3 0}$. 与此同时, 可见光照 射下芳基重氮酯产生的游离态卡宾能对 30 进行 $\mathrm{O}-\mathrm{H}$ 插入得到最终的三取代羟胺产物. 尝试了亚硝基苯以及 其他类型的亲核试剂, 反应未能顺利进行. 在这些反应 中, 亚硝基苯作为卡宾捕获试剂的反应速率远快 于亚硝基苯与亲核试剂的亚硝基-aldol 反应速率.

上述讨论的可见光促进卡宾中间体对 $\mathrm{X}-\mathrm{H}$ 键的插 入反应大多局限在一步反应过程. 近期，我们通过反应 的合理设计, 将 $\mathrm{X}-\mathrm{H}$ 插入反应后所得到的产物原位进 行后续反应，实现了一些重要的有机结构片段的构建. 选择邻羟基苯基取代的对醌类化合物 31 ( $p$-QMs)作为

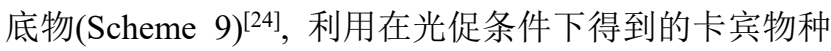
首先对化合物 31 进行 $\mathrm{O}-\mathrm{H}$ 键插入得到中间产物 33, 随后在碱性条件下脱质子, 经过分子内的环化构建一系 列 2-取代苯并呋喃衍生物 32 $^{[25]}$. 实验证明, 当使用 $\mathrm{Cs}_{2} \mathrm{CO}_{3}$ 作碱, 室温下分步进行时, 该反应可以较高的产 率、中等的非对映选择性得到目标杂环化合物. 控制实 验表明 32 的生成并不是通过 $\mathbf{3 1}$ 与芳基重氮酯间的直接 $[4+1]$ 环加成过程.

选择性转化是有机合成中常见的问题之一，例如在 2-吡啶酮 34 的官能化中, $O$ 和 $N$ 的官能化通常是同时发 生. 近期, 西北师范大学杨靖亚、霍聪德课题组 ${ }^{[26]}$ 通过 利用芳基重氮酯 $\mathbf{1}$ 在可见光照射下产生的游离态卡宾作 为关键活性物种，选择性地实现了 2-吡啶酮 34 的 $\mathrm{O}$ 官 能化 (Scheme 10). 作者通过控制实验给出了以下两种 可能的反应机理: 第一种是在可见光促进下芳基重氮酯 1 光解产生游离态卡宾 2 , 随后加成到 2-吡啶酮中产生 自由基中间体 37, 最后通过㩲氢得到目标产物 35. 另外 一种情况作者认为可能是产生的游离态卡宾首先描取 2-吡啶酮 34 的互变异构体 36 中的氢, 产生自由基 38 和 39, 随后通过自由基-自由基耦合生成相应的 $\mathrm{O}$ 官能化 产物.

腙类化合物，尤其是对甲苯磺酰腙在碱性条件下可 以原位产生重氮化合物. 基于这一原理，四川大学吴勇 团队报道了可见光和碱促进的腙类化合物与芳胺的偶

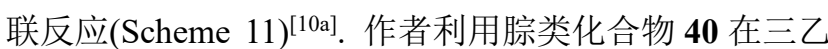
胺存在下原位生成重氮化合物 $\mathbf{1}$, 随后在光照下分解氮

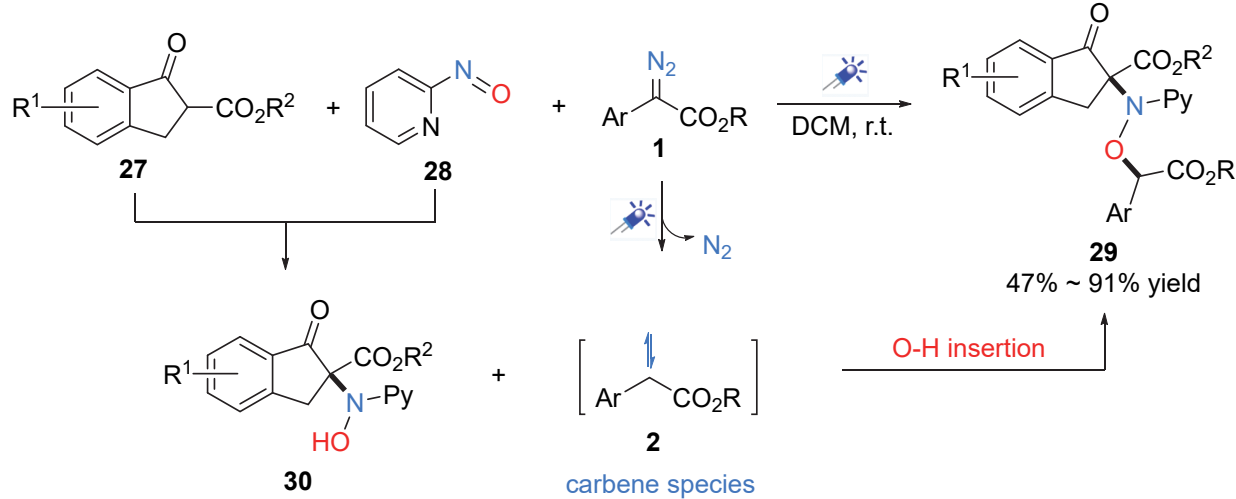

图式8 可见光促进的芳基重氮酯参与的多组分反应

Scheme 8 Visible light promoted multicomponent reactions involving aryldiazoacetates 


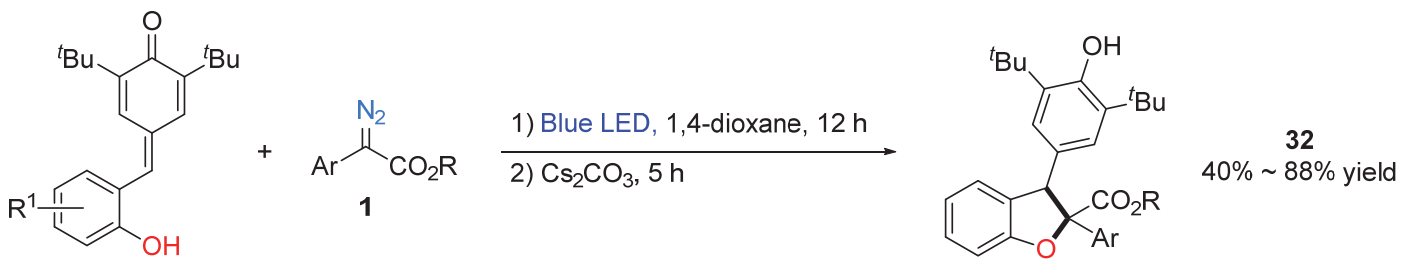

31

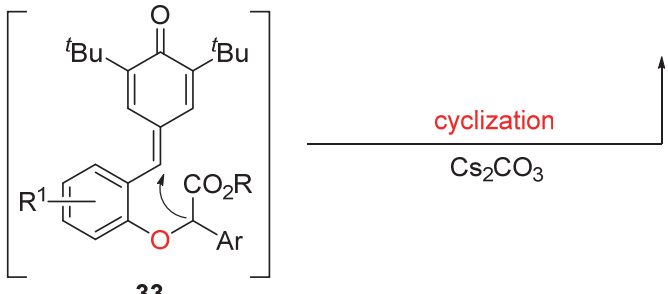

33

图式 9 可见光和碱促进的苯并呋喃衍生物的合成

Scheme 9 Visible light and base promoted synthesis of benzofuran derivatives

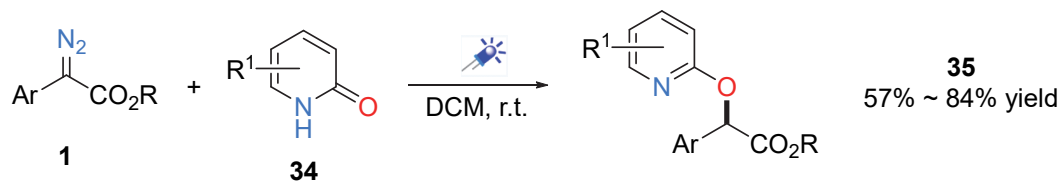

plausible reaction mechanism

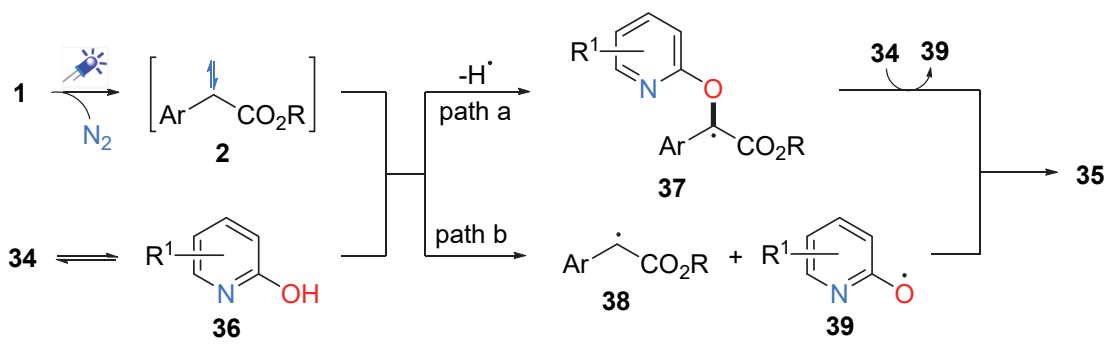

图式 10 可见光促进 2-吡定酮的 $O$-烷基化反应

Scheme 10 Visible light promoted $O$-alkylation 2-pyridinone

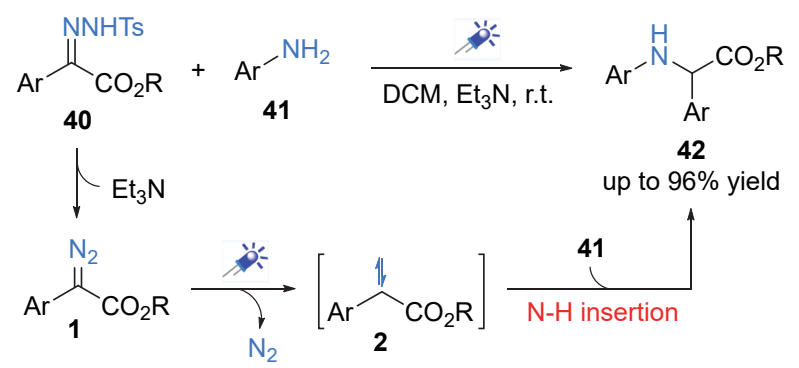

图式 11 可见光和碱促进的腙类化合物与芳胺的反应 Scheme 11 Visible light and base promoted reaction of hydrazone compounds with aromatic amines

气产生游离态卡宾中间体 2,2 对芳胺的 $\mathrm{N}-\mathrm{H}$ 键进行插 入反应得到最终的仲胺类产物 42. 随后, 作者发现该反 应在水中也能够顺利进行 ${ }^{[10 b]}$.

2020 年, 西北师范大学杨靖亚、马奔课题组报道了 可见光促进的芳基重氮酯与苯并三氮唑的选择性 $\mathrm{N}-\mathrm{H}$ 插入反应(Scheme 12) ${ }^{[27]}$. 实验过程中发现, 芳基重氮酯 仅在可见光照射下无法直接完成对苯并三氮唑 $\mathrm{N}^{1}$ 位的 插入, 而是需要使用额外的对苯醌作为催化剂. 该反应
中, 苯并三唑氮自由基稳定性差异是实现该反应 $\mathrm{N}^{1}$ 选 择性控制的关键因素之一. 作者提出了以下可能的机理 来解释反应的选择性. 首先, 在可见光的照射下, 对苯 醌被激发到具有良好篗氢能力的激发态 $\mathbf{P B Q}$. 然后 $P B Q *$ 与苯并三氮唑 43 通过氢原子转移(HAT)或质子耦 合电子转移(PCET)产生自由基中间体 45 和半醌自由基 46. 芳基重氮酯在可见光的照射下光解产生的游离态卡 宾 2 被自由基中间体 45 捕获产生新的碳中心自由基 47 . 最终碳中心自由基 47 与半醌自由基通过氢原子转移 (HAT) 获得最终目标产物 44, 完成苯醌催化剂的催化循 环. 最近, Sen 课题组 ${ }^{[28]}$ 也实现了光促条件下芳基重氮 酯对吲哚 $\mathrm{N}-\mathrm{H}$ 的插入, 并且在连续流动光化学的条件 下依然可以顺利进行.

对于氨基酸或者多肽的选择性修饰是生物化学以 及药物化学中需要解决的关键科学问题. 2020 年, 南京 工业大学邱江凯、郭凯等报道了可见光促进的芳基重氮 酯 1 对半胱氨酸骨架 48 中的 $\mathrm{S}-\mathrm{H}$ 键的选择性插入反应 (Scheme 13) ${ }^{[29]}$. 机理验证实验表明，反应可能是通过 

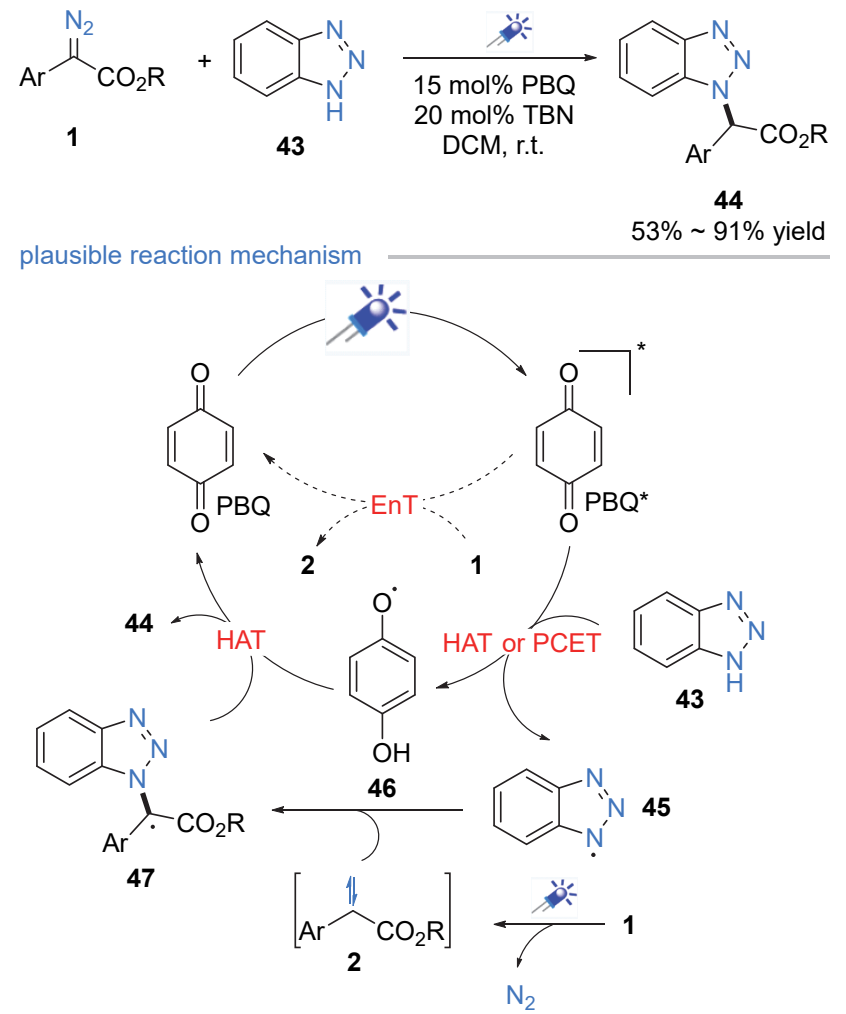

图式 12 可见光促进芳基重氮酯与苯并三唑的 $N^{1}$-烷基化反 应

Scheme 12 Visible light promoted $N^{1}$-alkylation of aryldiazoacetates with benzotriazoles

$$
1
$$

图式 13 可见光促进 $\mathrm{S}-\mathrm{H}$ 键的插入反应

Scheme 13 Visible light $\mathrm{S}-\mathrm{H}$ bond insertion reaction

自由基的途径进行的. 同时作者也通过流动光化学的手 段证明了该反应的实用性. 值得注意的是, 四川大学吴 勇课题组 ${ }^{[10]}$ 以及西北师范大学杨靖亚与霍聪德课题 组 ${ }^{[30]}$ 也报道了可见光促进的重氮化合物或腙类化合物 对芳基硫酚的 $\mathrm{S}-\mathrm{H}$ 键插入反应.

\section{3 叶立德的形成及后续反应}

\section{1 通过硫叶立德中间体}

Doyle-Kirmse 反应被发现已有 50 余年，也是[2,3]$\sigma$ 重排的代表性反应之一 ${ }^{[31]}$. 在可见光促进的游离态卡 宾转化反应兴起之前, Doyle-Kirmse 反应均以金属催化 为主. 2019 年, Koenigs 课题组 ${ }^{[32]}$ 报道了可见光促进的芳 基重氮酯 1 和烯丙基硫化物 $\mathbf{5 0}$ 在无任何催化剂以及添 加剂作用下的 Doyle-Kirmse 反应(Scheme 14). 作者也通
过流动光化学的手段对 Doyle-Kirmse 反应产物 51 进行 大量合成，并且反应收率并没有明显的下降.

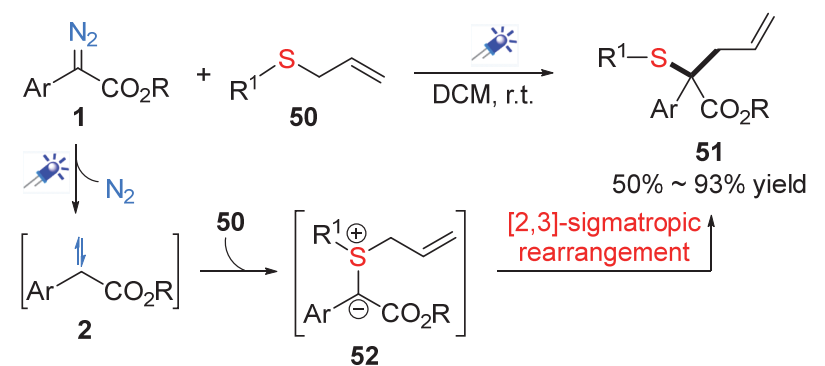

图式 14 可见光促进的 Doyle-Kirmse 反应

Scheme 14 Visible light promoted Doyle-Kirmse reaction

二氟亚甲基 $\left(\mathrm{CF}_{2}\right)$ 是非常有用的含氟片段，广泛应 用于功能材料、农药和药物化学等领域 ${ }^{[33]}$. 昆明理工大 学江玉波、肖铁波团队 ${ }^{[34]}$ 通过光促 Doyle-Kirmse 反应合 成了一系列含有二氟亚甲基 $\left(\mathrm{CF}_{2}\right)$ 片段的烯烃化合物 $\mathbf{5 4}$ (Scheme 15). 在该反应中，二氟烯丙基硫化物 53 可以 捕获芳基重氮酯光解产生的游离态卡宾，随后经过 [2,3]-sigmatropic 重排以中等至良好的收率获得目标产 物 54. 此外, 在该项工作中二氟烯丙基硫化物 53 以及 芳基重氮酯 1 都表现出很好的官能团耐受性.

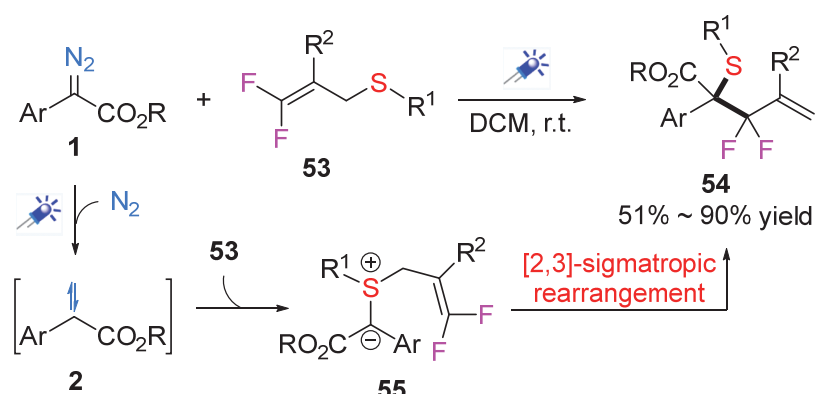

图式 15 可见光促进芳基重氮酯与二氟烯丙基硫化物的反应 Scheme 15 Visible light promoted reaction of aryldiazoacetates with difluoroallyl sulfides

亚砜叶立德作为一种高效的 $\mathrm{C} 1$ 合成子, 广泛应用 于多种重要的有机转化过程中，同时关于硫叶立德合成 方法学的研究也颇受关注 ${ }^{[35]}$. 近期, 我们课题组 ${ }^{[36]}$ 报道 了可见光促进的芳基重氮酯与亚砜 56 的反应来构建一 系列亚砜叶立德产物 57 (Scheme 16). 该反应只需使用 可见光进行照射, 不需要任何催化剂以及添加剂就能以 中等至良好的收率获得目标产物 $\mathbf{5 7}$, 同时该策略也表 现出了良好的官能团耐受性. Bhat 课题组 ${ }^{[37]}$ 发现, 在可 见光照射下，通过添加 $\mathrm{DABCO}$ 能够更高效地促进该反 应的顺利进行.

最近, 浙江大学王彦广课题组 ${ }^{[38]}$ 发现在可见光照 射下 4-重氮-1,4-二氢异喹啉-3-酮(58)光解产生的游离态 卡宾，可以被烯丙基/炔丙基硫化物捕获，生成硫叶立德 


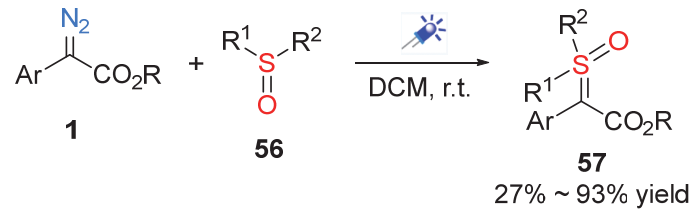

图式 16 可见光促进亚砜叶立德的合成反应 Scheme 16 Visible light promoted synthesis of sulfoxonium ylides

中间体 61, 最后通过[2,3]-sigmatropic 重排合成了一系 列含有烯烃或者联烯结构的硫化物 60 (Scheme 17). 值 得注意的是, 该反应所用的重氮化合物不再局限于之前 常用的芳基重氮酯, 这为日后扩大可见光促进重氮化合 物转化的底物范围起到了一定的促进作用.

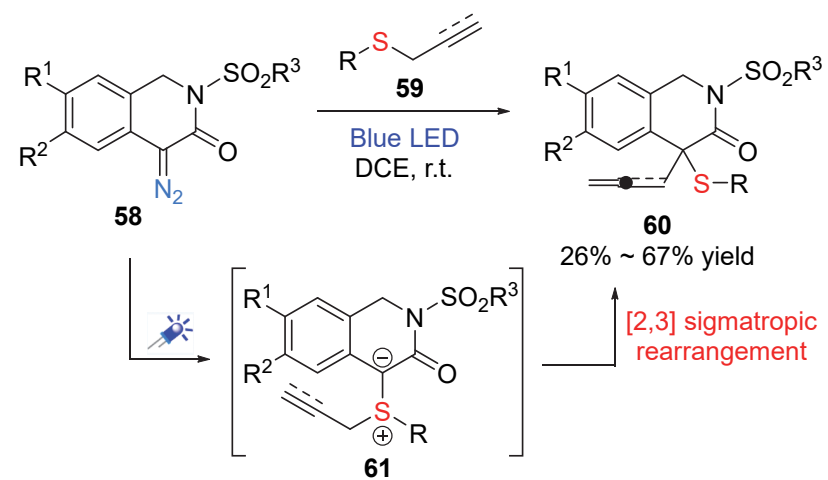

图式 17 可见光促进 4-重氮-1,4-二氢异喹啉-3-酮与烯丙基/ 炔丙基硫化物的反应

Scheme 17 Visible light promoted reaction of 4-diazo-1,4dihydroisoquinolin-3-ones with allyl/propargyl sulfide

\section{2 通过氧叶立德中间体}

由于二氧化碳在自然界中天然丰度高、无毒、可再 生等优势, 已经逐渐成为有机合成中极具吸引力的碳 源. 近期, 华南理工大学墄朝荣和江焕峰课题组 ${ }^{[39]}$ 利用 芳基重氮酯 $\mathbf{1}$ 在可见光照射下与胺 19 和二氧化碳 62 反 应, 以中等至良好的收率合成一系列氨基甲酸酯 (Scheme 18). 实验过程中发现所得到的氨基甲酸酯结 构受溶剂影响较大, 当使用四氢呋喃作溶剂时, 溶剂将 会参与到反应过程中, 最终会获得四组分偶联产物 63 . 然而, 当使用混合溶剂 1,4 -二氧六环/乙腈时, 将得到重 氮化合物 1 、胺 19 和二氧化碳 62 的三组分偶联反应. 值 得注意的是, 无论反应在何种溶剂中进行, 都只需要使 用 $101 \mathrm{kPa}$ 二氧化碳, 并且在室温条件下即可进行. 同 时作者也提出了以下可能的反应机理: 首先, 氨基甲酸 根阴离子 65 和铵盐 66 很容易在室温下由胺 19 和二氧 化碳 62 产生, 当使用 1,4-二氧六环/乙腈作为反应溶剂 时, 光照条件下产生的游离态卡宾 $\mathbf{2}$ 会立即被 $\mathbf{6 5}$ 所捕 获, 产生两性离子中间体 67 , 随后经过质子转移获得最 终的氨基甲酸酯 64. 当使用四氢呋喃作为反应溶剂时,
游离态卡宾 2 会优先捕获四氢呋喃形成氧鎓叶立德中间 体 68, 随后氨基甲酸根阴离子 65 作为亲核试剂促进四 氢呋喃开环，最后经过质子化得到四组分偶联产物 63 .

我们课题组在探索芳基重氮酯 1 与肜 25 反应时，也 发现如果使用四氢呋喃作为溶剂, 光解产生的游离态卡 宾首先被四氢呋喃捕获, 产生氧鎓叶立德中间体 68 , 随 后肜作为亲核试剂促进四氢呋喃开环，最终生成三组分 偶联产物 70 (Scheme 19) ${ }^{[20]}$.

\section{3 通过氮叶立德中间体}

酰胺键是自然界中最重要的结构单元之一, 大约四 分之一的上市药物和三分之二的候选药物中至少含有 一个酰胺键 ${ }^{[40]}$. 胺和羧酸的直接缩合是构建酰胺键最 直接的方法, 因为原料易得且价格低廉, 然而这种方法 需要使用化学计量的活化试剂, 原子经济性较差. 2020 年, 我们课题组 ${ }^{[41]}$ 报道了可见光促进的芳基重氮酯 $\mathbf{1}$ 与 亚硝基芳烃 72 通过硝酮的原位产生/重排构建酰胺键的 方法(Scheme 20). 在可见光的照射下, 芳基重氮酯光解 产生游离态卡宾 2, 随后亚硝基芳烃通过其亲核的 N-中 心与卡宾反应生成两性离子中间体 $\mathbf{7 5}$, 它将进一步转 化为更稳定的硝酮 73. 实验结果表明, 硝酮中的大共轭 基团可以继续吸收可见光, 促进其进一步环化成氮氧环 丙烷中间体 76, 随后重排成最终的酰胺产物 74. 理论计 算表明, 74生成的 Gibbs 自由能比苯基迁移产物的 Gibbs 自由能低 $27.9 \mathrm{~kJ} / \mathrm{mol}$, 表明酯基迁移优于苯基迁移. 值 得一提的是，也可以通过反应时间的控制来合成具有高 应用价值的硝酮产物 $\mathbf{7 3}^{[42]}$. 最近 Chatterjee 课题组 ${ }^{[43]}$ 也 发现, 使用芳基重氮酯与亚硝基化合物在二氯甲烷中进 行光照, 可以选择性地合成 $\alpha$-酮酸酯.

有机含氮杂环化合物广泛存在于天然生物碱、合成 药物以及功能材料中. 近期, 使用六氢-1,3,5-三嗪 77 作 为卡宾捕获试剂, 实现了两类有机含氮杂环化合物的可 控合成(Scheme 21) ${ }^{[44]}$. 在可见光的照射下, 可以通过溶 剂的不同来选择性地高效合成氮杂环丙烷 78 和咪唑啉 79. 通过控制实验和密度泛函理论(DFT)计算提出了可 能的反应机理. 对于在二氯甲烷(DCM)中合成的咪唑啉 79, 首先六氢-1,3,5-三嗪 77 作为卡宾捕获试剂来捕获重 氮化合物光解产生的游离态卡宾 $\mathbf{2}$, 再经过开环产生两 性离子中间体 81, 随后经过 $\mathrm{C}-\mathrm{N}$ 键的旋转以及分子内 的取代获得最终 $[4+1]$ 产物咪唑啉 79, 同时释放出一分 子亚胺 83. 对于在二甲亚砜(DMSO)中合成的氮杂环丙 烷，考虑到溶剂效应，有以下两种可能的反应机理：六 氢-1,3,5-三嗪 77 在 DMSO 中分解出相应的亚胺 83, 随 后亚胺作为卡宾捕获试剂, 再经过分子内的环化得到 [2 +1 ]环加成产物 78 (Scheme 21, path a). 另外一种情况 可能依然是六氢-1,3,5-三嗪 77 作为卡宾捕获试剂产生 


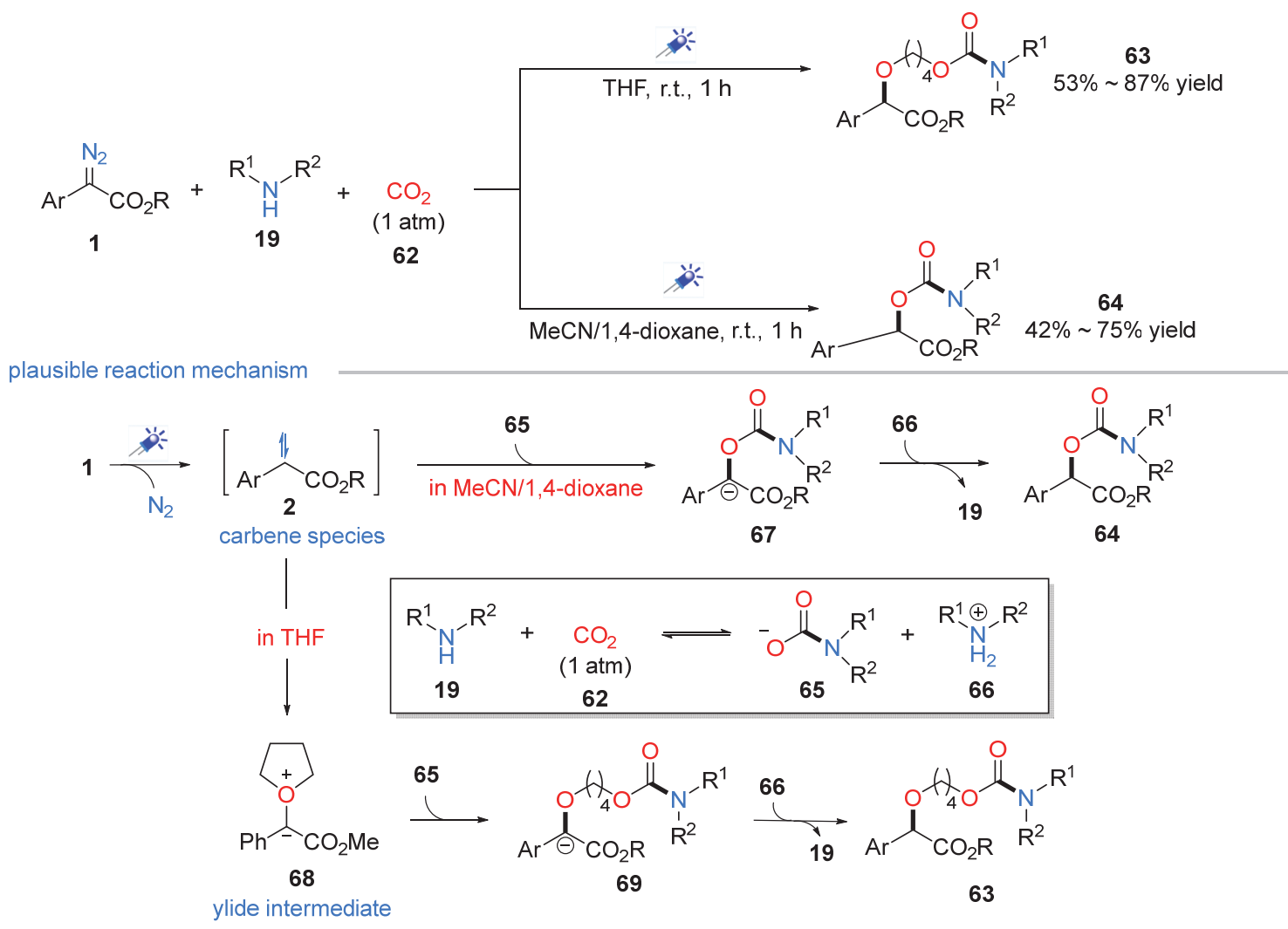

图式 18 可见光诱导芳基重氮酯、胺、二氧化碳参与的多组分反应

Scheme 18 Visible light induced multicomponent reactions of aryldiazoacetates, amines and carbon dioxide

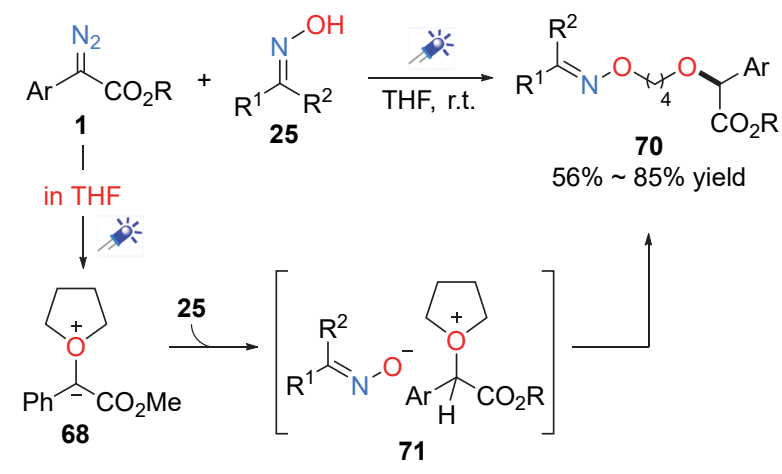

图式 19 可见光促进芳基重氮酯、肜和四氢呋喃的多组分反 应

Scheme 19 Visible light promoted multicomponent reaction of aryldiazoacetates, oximes and tetrahydrofurans

两性离子中间体 $\mathbf{8 1}$, 随后经过分子内的环化以及两分 子的亚胺离去获得目标氮杂环丙烷产物 78 (Scheme 21, path b).

2020 年, 中山大学徐新芳和胡文浩课题组 ${ }^{[45]}$ 报道 了可见光促进的芳基重氮酯 1 与邻胺基苯乙酮 85 形式 上的 $[4+1]$ 环加成反应(Scheme 22). 该反应为多取代吲 哚啉衍生物 86 的光化学合成提供了一条新型有效的方 法. 总体而言, 反应能够以良好的收率以及非对映选择 性得到目标杂环产物. 作者结合 DFT 计算给出了可能
的反应机理，首先光促产生的游离态卡宾被邻胺基苯乙

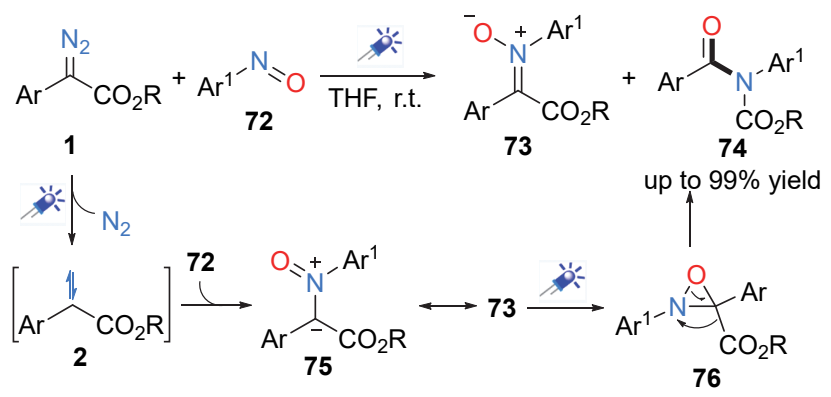

图式 20 可见光促进的硝酮原位产生/重排构建酰胺键 Scheme 20 Visible light promoted in situ generation/ rearrangement of nitrones to construct amide bonds

酮 85 捕获产生叶立德中间体 87 , 随后通过质子转移形 成的 $(E)$-烯醇物种 88 是该转化的关键中间体，最终通过 一个 Aldol 类型的加成反应得到最终目标产物 86.

\section{4 其他类型反应}

芳基重氮酯类型的重氮化合物在可见光照射下会 光解产生游离态卡宾 2 , 然而只有吸电子取代基的重氮 化合物能在可见光照射下保持不变. 中山大学周否课题 组 ${ }^{[6 b]}$ 利用两种重氮化合物的这一区别, 发展了一类三 取代烯烃合成的新反应(Scheme 23). 作者利用 Donor- 


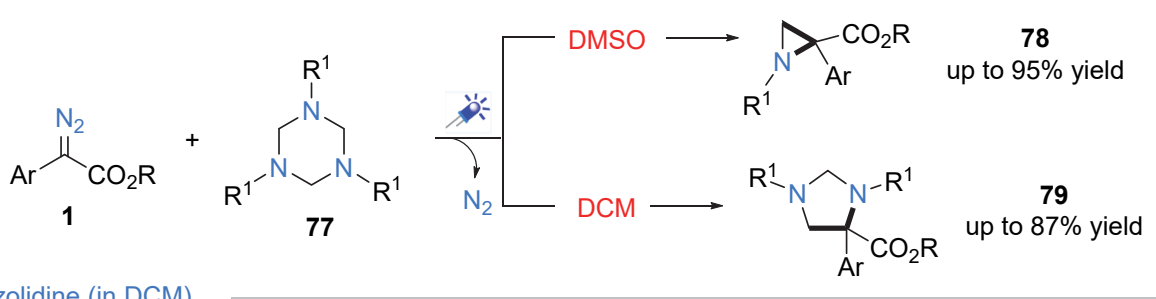

Formation of imidazolidine (in DCM)

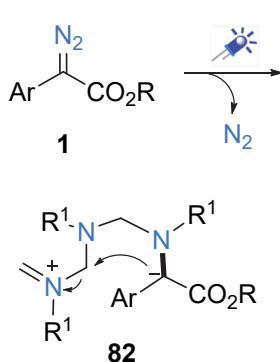

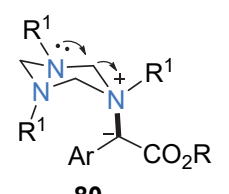

80

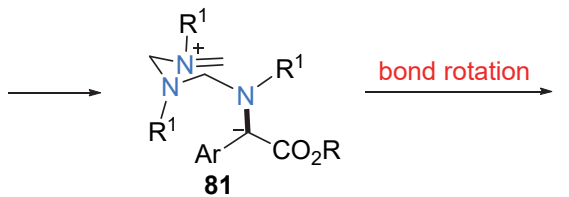<smiles>[R1]CC1(O)CN([R])CN1[R]</smiles>

Formation of aziridine (in DMSO) path a:

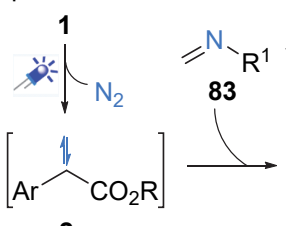

2<smiles>[R]OC(=O)C1([Al])CC2CC[N+]21[R17]</smiles>
77<smiles>[R]OC([Te])C1([18O])CN1[R]</smiles>

path b:

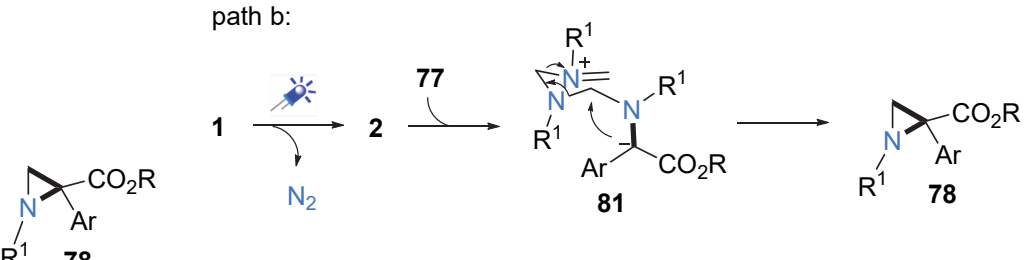

图式 21 可见光促进氮杂环丙烷和咪唑啉的选择性合成

Scheme 21 Selective synthesis of aziridine and imidazolidine promoted by visible light

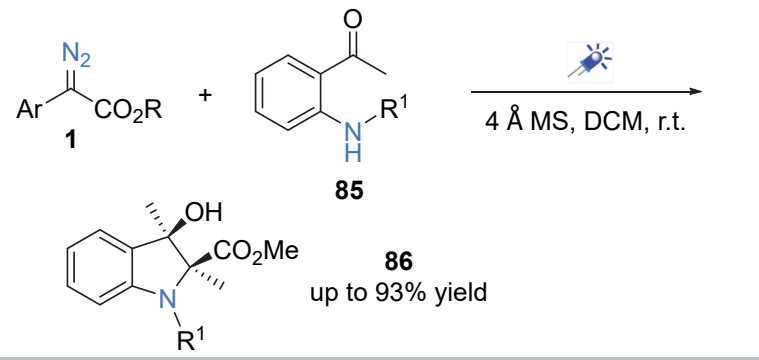

via<smiles>[R]OC(=O)C(Br)N([R7])c1ccccc1C(C)=O</smiles>

ylide intermediate

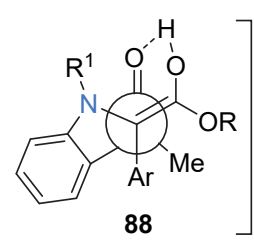

(E)-enol species
图式 22 可见光促进的芳基重氮酯与邻胺基苯乙酮的反应 Scheme 22 Visible light promoted reaction of aryldiazoacetates with $o$-aminoacetophenones

Acceptor 类型的芳基重氮酯 $\mathbf{1}$ 光解产生的游离态卡宾, 利用另一分子稳定的重氮化合物 89 卡宾捕获试剂, 产 生两性离子中间体 91, 随后经过氮气消除实现三取代 烯烃 90 的光化学合成. 此外, 该反应不仅具有很高的收 率, 而且选择性也很好, 绝大多数烯烃产物的顺/反比均 超过了 1：20. 近期, 使用硫叶立德来代替重氮化合物 89, 同样实现了三取代烯烃的高效高选择性合成 ${ }^{[46]}$.

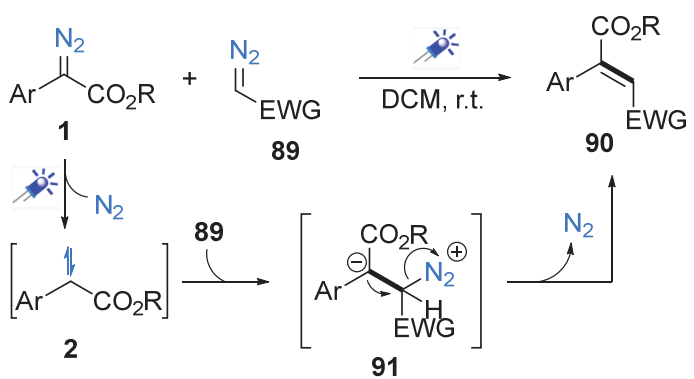

图式 23 可见光促进重氮化合物的交叉偶联反应 Scheme 23 Visible light promoted coupling of diazo compounds

\section{5 总结与展望}

从上述反应可以看出, 与传统的过渡金属催化的重 氮化合物转化反应相比，可见光促进的重氮化合物通过 游离态卡宾的转化具有很多优势, 例如: 仅需要使用可 见光作为绿色能源，室温进行，反应无需严格的除水除 氧等繁琐操作等. 自 2018 年 Davies 课题组和周磊课题 组分别独立报道了芳基重氮酯在可见光照射下能有效 地光解生成游离态卡宾物种以来, 可见光照射下重氮化 合物的转化反应受到了越来越多科研工作者的关注，也 催生了许多绿色高效的有机合成新反应. 其反应模式也 从简单重复已知过渡金属催化过程，发展到设计新的多 步或多组分反应以构建更为复杂的有机分子. 
虽然该领域在过去的几年里取得了一些进展, 但挑 战和不足依然存在. 首先, 该类型反应的底物大多局限 于使用 Donor-Acceptor 类型的重氮化合物, 且以芳基重 氮酸酯为主, 高效开发其他类型的重氮化合物, 进一步 丰富反应底物类型是该领域未来需要努力的第一个方 向. 其次, 由于卡宾的反应活性高, 如何实现可见光照 射下重氮化合物的催化不对称转化, 是科研工作者需要 思考并解决的另一个问题. 另外, 从机理上说, 光促重 氮化合物通过游离态卡宾的转化的反应特性与传统的 金属催化相比, 有很大的不同, 很多反应的详细机理有 待于进一步完善和探索.

\section{References}

[1] Meerwein, H.; Rathjen, H.; Werner, H. Ber. Dtsch. Chem. Ges. 1942, 75, 1610 .

[2] Kirmse, W. Carbene Chemistry, Academic Press, New York, 1971, Vol. 1, pp. $9 \sim 84$.

[3] Skell, P. S.; Etter, R. M. Proc. Chem. Soc. London 1961, 443.

[4] (a) Ford, A.; Miel, H.; Ring, A.; Slattery, C. N.; Maguire, A. R.; McKervey, M. A. Chem. Rev. 2015, 115, 9981.

(b) Xia, Y.; Qiu, D.; Wang, J. Chem. Rev. 2017, 117, 13810.

(c) Liu, L.; Zhang, J.-L. Chin. J. Org. Chem. 2017, 37, 1117 (in Chinese).

(刘路, 张俊良, 有机化学, 2017, 37, 1117.)

(d) Yan, G.; Kuang, C.; Peng, C.; Wang, J. Chin. J. Org. Chem. 2009, 29, 813 (in Chinese).

(严国兵，匡春香，彭程，王剑波，有机化学, 2009, 29, 813.)

(e) Tang, M.; Xing, D.; Cai, M.; Hu, W. Chin. J. Org. Chem. 2014, 34, 1268 (in Chinese)

(唐敏，邢栋，蔡茂强，胡文浩，有机化学, 2014, 34, 1268.)

[5] (a) Xuan, J.; Xiao, W.-J. Angew. Chem., Int. Ed. 2012, 51, 6828.

(b) Prier, C. K.; Rankic, D. A.; MacMillan, D. W. C. Chem. Rev. 2013, 113, 5322.

(c) Chen, Y.; Lu, L.-Q.; Yu, D.-G.; Zhu, C.-J.; Xiao, W.-J. Sci. China Chem. 2019, 62, 24.

(d) Cai, B.-G.; Xuan, J.; Xiao, W.-J. Sci. Bull. 2019, 64, 337.

(e) Xuan, J.; He, X.-K.; Xiao, W.-J. Chem. Soc. Rev. 2020, 49, 2546.

[6] (a) Jurberg, I. D.; Davies, H. M. L. Chem. Sci. 2018, 9, 5112.

(b) Xiao, T.; Mei, M.; He, Y.; Zhou, L. Chem. Commun. 2018, 54, 8865 .

[7] Jana, S.; Pei, C.; Empel, C.; Koenigs, R. M. Angew. Chem., Int. Ed. 2021, 60, 13271.

[8] Ciszewski, Ł. W.; Rybicka-Jasińska, K.; Gryko, D. Org. Biomol. Chem. 2019, 17, 432.

[9] (a) Yang, Z.; Stivanin, M. L.; Jurberg, I. D.; Koenigs, R. M. Chem. Soc. Rev. 2020, 49, 6833.

(b) Durka, J.; Turkowska, J.; Gryko, D. ACS Sustainable Chem. Eng. 2021, 9, 8895.

[10] (a) Xu, Y.; Lv, G.; Yan, K.; He, H.; Li, J.; Luo, Y.; Lai, R.; Hai, L.; Wu, Y.; Chem. Asian J. 2020, 15, 1945.

(b) Yan, K.; He, H.; Li, J.; Luo, Y.; Lai, R.; Guo, L.; Wu, Y. Chin. Chem. Lett. 2021, DOI:10.1016/j.cclet.2021.05.031.

[11] Guo, Y.; Nguyen, T. V.; Koenigs, R. M. Org. Lett. 2019, 21, 8814.

[12] Zhao, S.; Cheng, X.-X.; Gao, N.; Qian, M.; Chen, X. J. Org. Chem. 2021, 86, 7131.

[13] Reiser, O. Isr. J. Chem. 2016, 56, 531.

[14] (a) Empel, C.; Koenigs, R. M. J. Flow Chem. 2020, 10, 157. (b) Hommelsheim, R.; Guo, Y.; Yang, Z.; Empel, C.; Koenigs, R. M. Angew. Chem., Int. Ed. 2019, 58, 1203.

[15] Klöpfer, V.; Eckl, R.; Floß, J.; Roth, P. M. C.; Reiser, O.; Barham, J. P. Green Chem. 2021, 23, 6366.

[16] Guha, S.; Gadde, S.; Kumar, N.; Black, D. S.; Sen, S. J. Org. Chem. 2021, 86, 5234.

[17] Ford, A.; Miel, H.; Ring, A.; Slattery, C. N.; Maguire, A. R.; McKervey, M. A. Chem. Rev. 2015, 115, 9981.

[18] (a) He, F.; Li, F.; Koenigs, R. M. J. Org. Chem. 2020, 85, 1240. (b) He, F.; Koenigs, R. M. Chem. Commun. 2019, 55, 4881.

(c) Stivanin, M. L.; Fernandes, A. A. G.; Silva, A. F.; Okada Jr, C. Y.; Jurberg, I. D. Adv. Synth. Catal. 2020, 362, 1106.

[19] Itsuno, S.; Matsumoto, T.; Sato, D.; Inoue, T. J. Org. Chem. 2000, 65,5879 .

[20] Qian, L.; Cai, B.-G.; Li, L.; Xuan, J. Org. Lett. 2021, 23, 6951.

[21] Lu, F.-D.; He, G.-F.; Lu, L.-Q.; Xiao, W.-J. Green Chem. 2021, 23, 5379.

[22] Cai, B.-G.; Chen, Z.-L.; Xu, G.-Y.; Xuan, J.; Xiao, W.-J. Org. Lett. 2019, 21, 4234.

[23] Cai, B.-G.; Li, Q.; Zhang, Q,; Li, L.; Xuan, J. Org. Chem. Front. 2021, 8, 5982.

[24] (a) Zhou, S.-J.; Cheng, X.; Hu, C.-X.; Xu, G.-Y.; Xiao, W.-J.; Xuan, J. Sci. China Chem. 2021, 64, 61.

(b) Cheng, X.; Zhou, S.-J.; Xu, G.-Y.; Wang, L.; Yang, Q.-Q.; Xuan, J. Adv. Synth. Catal. 2020, 362, 523.

[25] Zhou, S.-J.; Cai, B.-G.; Hu, C.-X.; Cheng, X.; Li, L.; Xuan, J. Chin. Chem. Lett. 2021, 32, 2577.

[26] Yang, J.; Wang, G.; Zhou, H.; Li, Z.; Ma, B.; Song, M.; Sun, R.; Huo, C. Org. Biomol. Chem. 2021, 19, 394.

[27] Yang, J.; Duan, J.; Wang, G.; Zhou, H.; Ma, B.; Wu, C.; Xiao, J. Org. Lett. 2020, 22, 7284.

[28] Maiti, D.; Das, R.; Sen, S. J. Org. Chem. 2021, 86, 2522.

[29] Qian, L.-Z.; Yan, X.; Cui, Y.-S.; Sun, Q.; Duan, X.; Zhuang, K.-Q.; Chen, L.; Qiu, J.-K.; Guo, K. Adv. Synth. Catal. 2020, 362, 5093.

[30] Yang, J.; Wang, G.; Chen, S.; Ma, B.; Zhou, H.; Song, M.; Liu, C.; Huo, C. Org. Biomol. Chem. 2020, 18, 9494.

[31] Kirmse, W.; Kapps, M. Chem. Ber. 1968, 101, 994

[32] Hommelsheim, R.; Guo, Y.; Yang, Z.; Empel, C.; Koenigs, R. M. Angew. Chem., Int. Ed. 2019, 58, 1203.

[33] O'Hagan, D. Chem. Soc. Rev. 2008, 37, 308.

[34] Yang, J.; Wang, J.; Huang, H.; Qin, G.; Jiang, Y.; Xiao, T. Org. Lett. 2019, 21, 2654.

[35] Wang, Z.; Xu, X.; Kwon, O. Chem. Soc. Rev. 2014, 43, 2927.

[36] Lu, J.; Li, L.; He, X.-K.; Xu, G.-Y.; Xuan, J. Chin. J. Chem. 2021, 39, 1646.

[37] Khade, V. V.; Thube, A. S.; Warghude, P. K.; Bhat, R. G. Tetrahedron Lett. 2021, 77, 153258

[38] Xie, J.; Suleman, M.; Wang, Z.; Mao, X.; Mao, B.; Fan, J.; Lu, P.; Wang, Y. Org. Biomol. Chem. 2021, 19, 6341.

[39] Cheng, R.; Qi, C.; Wang, L.; Xiong, W.; Liu, H.; Jiang, H. Green Chem. 2020, 22, 4890 .

[40] Crespo, L.; Sanclimens, G.; Pons, M.; Giralt, E.; Royo, M.; Albericio, F. Chem. Rev. 2005, 105, 1663.

[41] Cai, B.-G.; Luo, S.-S.; Li, L.; Li, L.; Xuan, J.; Xiao, W.-J. CCS Chem. 2020, 2, 2764.

[42] Cai, B.-G.; Li, L.; Xu, G.-Y.; Xiao, W.-J; Xuan, J. Photochem. Photobiol. Sci. 2021, 20, 823.

[43] Roy, S.; Kumar, G.; Chatterjee, I. Org. Lett. 2021, 23, 6709.

[44] Cheng, X.; Cai, B.-G.; Mao, H.; Lu, J.; Li, L.; Wang, K.; Xuan, J. Org. Lett. 2021, 23, 4109.

[45] Chen, J.; Liu, S.; Lv, X.; Hong, K.; Lei, J.; Xu, X.; Hu, W. J. Org. Chem. 2020, 85, 13920

[46] Ye, C.; Cai, B.-G.; Lu, J.; Cheng, X.; Li, L.; Pan, Z.-W. Xuan, J. J. Org. Chem. 2021, 86, 1012. 